\title{
tic\&société
}

Vol. 8, N 1-2 | 1er semestre 2014 et 2ème semestre 2014

Quelles perspectives critiques pour aborder les TIC ?

\section{Quelles perspectives critiques pour aborder les TIC ?}

Éric GEORGE

\section{Q OpenEdition \\ 1 Journals}

\section{Electronic version}

URL: http://journals.openedition.org/ticetsociete/1365

DOI: 10.4000/ticetsociete.1365

\section{Publisher}

Association ARTIC

\section{Electronic reference}

Éric GEORGE, «Quelles perspectives critiques pour aborder les TIC ? », tic\&société [Online], Vol. 8, N

1-2 I 1er semestre 2014 et 2ème semestre 2014, Online since 31 May 2014, connection on 19 April 2019. URL : http://journals.openedition.org/ticetsociete/1365 ; DOI : 10.4000/ticetsociete.1365 
tic\&société - 8 (1-2), 2014

\section{Quelles perspectives critiques pour aborder les TIC ?}

Éric GEORGE

École des médias

CRICIS

UQAM

george.eric@uqam.ca 


\title{
Quelles perspectives critiques pour aborder les TIC ?
}

\begin{abstract}
Éric George est détenteur d'un doctorat en communication de l'Université du Québec à Montréal (UQAM) et d'un doctorat en Sciences de l'information et de la communication de l'École Normale Supérieure de Paris obtenus en 2001. II est actuellement professeur titulaire à l'École des médias (Faculté de communication) de I'UQAM et directeur du Centre de recherche interuniversitaire sur la communication, l'information et la société (CRICIS). Ses recherches portent sur l'économie des médias, les politiques de communication, la communication à l'ère de la mondialisation, les usages sociaux des TIC, l'espace public médiatique et plus fondamentalement sur les rapports entre communication, capitalisme et démocratie. II est l'auteur de plus de quatre-vingt textes (articles, chapitres de livres et actes de colloques) avec révision par les pairs et, récemment, a été éditeur de trois publications: Critique, sciences sociales et communication (Mare et Martin, 2014) (avec Fabien Granjon); The Francophone School of Communication and Information (Canadian Journal of Communication, 2014), Autochtones et médias (Recherches amérindiennes au Québec, 2012).
\end{abstract}

Résumé : Les TIC jouant un rôle croissant tant à l'échelle planétaire que dans nos vies quotidiennes, les aborder d'un point de vue critique devient un enjeu scientifique majeur. Adoptant nous-même une telle démarche, nous nous interrogeons tout d'abord sur les sens à attribuer au terme "critique " et sur les significations de l'acronyme "TIC ». Après quoi, nous proposons de mettre l'accent sur plusieurs façons d'analyser les TIC d'un point de vue critique. Pour ce faire, nous commençons sur l'intérêt d'effectuer des analyses qui visent à replacer ces objets dans le contexte du "système technicien". Puis nous abordons deux autres ensembles de travaux, l'un consacré aux recherches en économie politique de la communication et l'autre portant sur les usages sociaux des TIC. Avant de conclure à la pertinence d'intégrer deux autres dimensions dans l'analyse : le temps long et les questions environnementales.

Mots-clés : TIC, critique, épistémologie, système technique, économie politique, usages, temps long, environnement. 
Abstract : As ICTs become more pervasive both globally and in our daily lives, investigating their roles and function from a critical perspective is a growing challenge. Adopting such an approach, we assess the meanings given to the term "critical" and to the acronym "ICT", and propose various ways in which to analyze ICTs through a critical lens. Our analysis begins by placing these objects in the context of the "technician system". We then discuss two other relevant bodies of literature; one dedicated to research in political economy of communication and the other to the social uses of ICTs. We conclude by noting the importance of integrating two additional dimensions into the analysis of this issue: temporality and environment.

Keywords : ICT, critique, epistemology, technical system, political economy, temporality, environment

Resumen : Las TIC desempeñan un creciente papel tanto a escala planetaria como en nuestra vida cotidiana y en consecuencia, el estudio de dichas tecnologías desde un punto de vista crítico se convierte en un reto científico fundamental. Desde esta perspectiva, en primer lugar se analizan los sentidos que pueden atribuirse al término "crítico" así como los significados del acrónimo TIC, para, a continuación, considerar las diversas formas de abordar las TIC, desde una perspectiva crítica. Para ello, se coloca el interés en efectuar análisis que tienden a considerar las TIC en un contexto de "sistema técnico ». Después se abordan otros dos tipos de trabajos : uno, consagrado a las investigaciones en economía política, y el otro a los usos sociales de las TIC. Se llega a la conclusión de que deben integrarse otras dimensiones en el análisis : el tiempo largo y la cuestión medioambiental.

Palabras clave : TIC, crítica, epistemología, sistema técnico, economía política, usos, tiempo largo, medio ambiente 
Nous intéresser aux perspectives critiques susceptibles d'analyser les TIC et leurs rapports avec le social nous apparaît d'autant plus crucial que celles-ci ont pris au cours des cent dernières années une place considérable dans toutes les activités de la vie quotidienne, ce qui s'explique par la dynamique à la fois d'ordre économique et technique qui permet dorénavant à l'être humain de commencer à penser sérieusement aussi bien à la possibilité de l'exode vers d'autres espaces planétaires qu'à la modification même de l'espèce à travers la manipulation de l'ADN ; et ce tout en pouvant également remettre en cause notre survie même dans l'écosystème qui nous a permis jusque là de déployer nos activités. Ainsi que le dit Jean-Guy Lacroix :

" entre autres avec la découverte de l'atome et l'invention de la bombe atomique, les rapports entre le vécu et le conçu, entre la capacité de faire et la volonté de faire, seront inversés [...]. Le vouloir-faire domine la capacité de faire, ce qui dote le sujet d'une liberté jamais jusqu'ici atteinte dans l'histoire de l'humanité. Cependant, ces mêmes nouvelles conditions de possibilité placent également le sujet devant des responsabilités d'une ampleur totalisante et d'un caractère plus fondamental que jamais auparavant. En effet, durant la dernière partie du $X X^{\mathrm{e}}$ siècle, la capacité d'action du sujet concerne l'écosystème dans sa totalité immédiate [...] et lui-même, comme totalité naturelle (le corps et l'espèce), et elle peut conduire tant à la préservation de l'écosystème qu'à sa destruction ou encore à la transformation de la nature même du vivant (p. ex., le génie génétique). 》 (1998, p. 87).

Nous sommes en effet à un moment charnière de l'histoire de la civilisation humaine dont nous devons prendre conscience (Morin, 2004) et les TIC y jouent de nombreux rôles qu'il importe d'analyser le plus complètement possible. Le présent texte vise à apporter en la matière une modeste contribution à partir de la mise en évidence de quelques orientations de recherche qui nous apparaissent soit toujours pertinentes, soit prometteuses.

\section{1. À propos du mot «critique » et de l'acronyme}

Nous interroger sur les rapports entre critique et TIC consiste tout d'abord à s'arrêter sur ce que nous entendons par ces deux termes, le langage constituant une «médiation par laquelle les individus agissent et interagissent dans le 
monde " pour reprendre la formule de John B. Thompson (1987, p. 7). En conséquence les idéologies circulent «sous forme d'énoncés, d'expressions, de mots prononcés ou écrits » (ibid., p. 8). Dans la perspective de Thompson, notre propos relève bien d'une interprétation du monde et le faire partager à travers cette publication consiste à le soumettre lui-même à la critique, notamment à celle de nos pairs.

À propos du terme "critique ", cela fait maintenant quatre ans que nous menons une réflexion à ce sujet qui a tout d'abord donné lieu à la tenue d'un séminaire mensuel du Centre de recherche interuniversitaire sur la communication, l'information et la société (CRICIS) en 2010 et 2011 sous le titre Pensée critique et communication, puis à celle d'un colloque international ayant pour intitulé Où (en) est la critique en communication ? (Kane et George, 2013). De l'ensemble des discours scientifiques, trois acceptations plus ou moins récurrentes lorsqu'il est question de pensée, de perspective, d'approche ou bien encore de regard critique, nous semblent à distinguer: (1) s'interroger en permanence sur la nature de la production des connaissances et sur notre rôle à ce sujet ; (2) reconnaître la nécessité de porter un regard exigeant sur notre monde tout en étant ouvert aux divers possibles susceptibles de favoriser un progrès dans l'humanisation de celui-ci ; (3) mettre l'accent sur les concepts qui nous apparaissent importants, tant en ce qui concerne les rapports de pouvoir que les possibilités en termes d'émancipation, et les " analyser » dans le cadre de divers contextes sociaux, à la fois macro et micro (George, 2014).

Lecteurs/trices pourront constater que dans les articles de ce numéro les regards portés sur les TIC mobilisent plus ou moins ces trois façons de concevoir la critique. Mais ne serait-il pas plus riche de les appréhender en complément l'une de l'autre ? En effet, penser de façon critique consiste à user de la raison avant tout, mais bien d'autres moyens d'expression, telle la création artistique, s'avèrent susceptibles de permettre cette pratique. De plus, pratiquer la raison est indissociablement lié à celle du doute systématique vis-à-vis d'illusions confortables ou de simplifications abusives. Un tel regard exige aussi de tenir compte du fait que la critique sociale ne repose plus uniquement sur le savoir d'une petite élite de la population, mais également sur les points de vue tant des individus que des collectifs plus ou moins organisés. Autrement dit, une nouvelle façon de faire consiste aussi à s'appuyer sur les mobilisations citoyennes. On pense par exemple au mouvement altermondialiste du début du troisième millénaire, mais également plus récemment au "printemps arabe " (notamment en Tunisie et en Égypte), aux mouvements des Indignados (Espagne) et Occupy! (en Amérique du Nord) ou encore au "printemps érable " au Québec. Reste à savoir - mais nous sommes ici aux frontières de notre texte - si ces mobilisations sont capables de renverser les rapports de 
pouvoir existants et de les remplacer par des relations plus égalitaires entre les composantes de l'ensemble de nos sociétés.

Voilà d'ailleurs que nous avons basculé de la première conception du terme " critique » à la deuxième. Comme le dit Étienne Balibar, " on ne peut vraiment entrer dans l'avenir qu'en faisant à chaque instant la critique minutieuse du passé " (1992, p. 13). Et celle-ci doit passer avant tout par l'analyse minutieuse de tous les rapports de pouvoir, à commencer par les rapports de classes, les rapports de genre et les rapports interculturels. Nous rejoignons ici Max Horkheimer (1996) qui considère que les chercheur/e/s critiques doivent prendre conscience que l'organisation sociale au sein de laquelle ils déploient leurs activités tend à être régie par la mise en valeur systématique du capital qui colonise une part toujours croissante de nos activités, et qu'il importe d'analyser toutes les conséquences de cet état de fait. Toutefois, et c'est ici qu'Horkheimer nous semble des plus pertinents, il importe tout autant de s'intéresser au "possible ", considéré comme immanent, celui-ci étant porté par des sujets sociaux dans des situations sociales historiquement déterminées.

À partir de cette prémisse, ne serait-il pas pertinent, voire nécessaire de réfléchir à la fois en termes de pouvoir, de rapports de force, d'exploitation, de domination, d'inégalités économiques et sociales, d'aliénation, mais aussi de résistance, d'émancipation, de changement social, de puissance d'agir ou bien encore d'empowerment (capacitation)? Ne faudrait-il pas combiner la mobilisation de plusieurs de ces concepts plutôt que de les traiter de façon séparée, d'aborder en tension permanente ceux qui portent en eux la reproduction du monde tel qu'il existe et ceux qui véhiculent des transformations positives, ce qui est de l'ordre des possibles que nous évoquions précédemment?

Ces questions étant posées au sujet du terme «critique », venons-en maintenant plus brièvement à l'expression «TIC». Le «T » renvoie le plus souvent au terme " technologies " mais ne devrions-nous pas plutôt parler de "techniques", le mot «technologie » renvoyant davantage aux discours (logos) sur la technique ? Cette première remarque étant faite, que recouvrent donc les TIC ? On pense à un vaste ensemble d'objets qui, au sein des secteurs de l'informatique, des télécommunications, de l'audiovisuel et bien entendu de l'internet, permettent d'accéder à des informations, de les stocker, de les transformer mais également de... communiquer. II y a donc derrière cet acronyme l'idée de manipuler des données, de jouer un rôle actif, ce qui nous renvoie à deux vocables, ceux d'« interactivité » et d'« interaction » (voir, entre autres, tic\&société, 2010 et antérieurement, plus spécifiquement Proulx et Sénécal, 1995). 
Mais il est maintenant parfois question de TNIC ou de TICN, le « $N$ » faisant référence au mot «numérique » qui renvoie à l'idée selon laquelle les informations de toutes sortes circulent de plus en plus facilement sous codage informatique binaire (les data), à travers des supports, écrans de toutes sortes ordinateurs, téléviseurs, tablettes, consoles de jeux vidéo et autres téléphones qualifiés d'intelligents - et les réseaux filaires ou non (satellites, micro-ondes, etc.) qui sont de plus en plus interconnectés. On pourrait d'ailleurs aussi qualifier ces outils de réseautiques puisque ceux-ci se développent en suivant cette morphologie comme le symbolise le "réseau des réseaux ». Un nombre toujours croissant de terminaux sont en effet de plus en plus systématiquement interconnectés les uns aux autres, tant et si bien que l'on en vient à parler de l'internet des objets ; ce qui renvoie justement à l'interconnexion d'artéfacts qui ne sont pas forcément a priori concernés par ces techniques d'information et de communication mais qui s'y retrouvent intégrés de facto. Toutefois, c'est le terme «numérique » qui semble s'être imposé, du moins pour un temps.

Reste maintenant à considérer que nous avons affaire à des techniques de l'information et de la communication. Par le passé, l'expression $\mathrm{Tl}$, plus employée, renvoyait expressément à l'idée que les objets dont il était question avaient avant tout pour objectif de manipuler de l'information. Nous n'étions pas loin dans certains cas d'une conception quelque peu cybernétique de l'information et d'ailleurs plusieurs auteur/e/s, à commencer par Manuel Castells (1998), ont tendance à reprendre l'acronyme TI. Pourtant, l'ajout du « C » et de facto du terme "communication» introduit une certaine complexité, les techniques dont il est question ne pouvant être résumées à de "simples " échanges d'information. Communiquer, n'est-ce pas «mettre en commun »? Dès lors, n'est-on pas dans le registre de l'intersubjectivité, de la perspective du vivre-ensemble, de la vie dans la cité (polis) dont il sera d'ailleurs question dans plusieurs textes de ce numéro ? Voilà, les termes du débat sont posés. Une analyse critique des TIC ne peut donc se faire sans s'intéresser aux critiques de la technique, mais les TIC, notamment de par leur dimension communicationnelle, ne sauraient être complètement considérées comme des «techniques » comme les autres.

\section{De la pertinence de quelques perspectives critiques sur les TIC}

\subsection{Penser la technique comme système}

L'idée forte selon laquelle les TIC font partie d'un ensemble plus vaste nous oriente vers des réflexions d'auteurs comme Jacques Ellul (1954, 1977, 1988) qui envisagent la technique comme système. Ce dernier attire notre attention 
sur le fait que la technique doit être analysée bien au-delà des usages. Celle-ci est devenue une finalité dans nos sociétés si l'on en croit Ellul qui estime d'ailleurs que "ce n'est pas la technique qui nous asservit mais le sacré transféré à la technique » (1973, p. 259). Plus rien n'est pensé sans technique et la technique va bien au-delà de l'objet lui-même. II serait donc vain d'analyser les TIC sans penser celles-ci comme des composantes d'un système technicien, nous dit-il, au service de valeurs telles que le progrès technique, l'efficacité ou la rationalisation. Ces valeurs seraient présentes tant dans les discours sur la technique que dans le design des interfaces des objets. Et en la matière, les concepteurs/trices seraient à la fois acteurs de l'innovation sociotechnique, mais également "agis par la technique ». Le mot même de technique ne renvoie donc plus seulement à de vastes ensembles d'objets, mais aussi à des "manières de faire". L'informatique peut elle-même être considérée comme une composante exemplaire du système technicien de Jacques Ellul au sens où il $\mathrm{y}$ a technique à partir du moment où il $\mathrm{y}$ a recherche de nouveaux moyens chargés d'augmenter l'efficacité.

L'exemple du développement du réseau internet est exemplaire à cet égard car si celui-ci a principalement reposé sur des motivations concernant les échanges et la coopération (Abbate, 1999), il l'a aussi été dans un souci d'être le plus efficace possible. La rationalité technique est donc toujours restée présente au cœur du projet. D'ailleurs, l'utilisateur actuel des TIC n'a-t-il pas tendance à correspondre à la figure d'un individu toujours plus calculateur, rationnel, visant la performance, la créativité, bref «entrepreneur de soi »? $\mathrm{Ne}$ tend-il pas à se transformer en investisseur ayant pour principale fonction de gérer des capitaux (économiques, culturels, intellectuels, réputationnels et humains) comme le dit Maxime Ouellet (2009, p. 146) ?

La proposition d'Ellul apparaît donc riche et stimulante. Toutefois, il a analysé la technique comme la caractéristique essentielle de notre époque en constatant que les sociétés capitalistes et communistes se ressemblaient de plus en plus fortement, ces deux projets accordant justement un poids central à la technique assez proche l'un de l'autre. Or, il est sans doute plus pertinent d'appréhender de pair systèmes technique et économique ; d'où notre intérêt pour la position d'Herbert Marcuse (1968). Ce dernier considère également que la technique forme un système mis en évidence quand on constate qu'au-delà des différentes utilisations qui en sont faites, son déploiement présente toujours les mêmes invariants. Les sociétés dites avancées imposent un type de rationalité qui fait appel à une dimension limitée du potentiel humain, "l'homme unidimensionnel » affirme-t-il. Mais si la technique est partout au point de former système, c'est non seulement parce que celle-ci, étant sacralisée, ne fait plus 
l'objet de critiques, c'est aussi parce qu'elle participe à l'accumulation du capital. À ce sujet, André Mondoux (2007) explique qu'on se retrouve avec deux ontologies, celle de la technique et celle du capitalisme. Le capitalisme utilise la technique à ses propres fins, mais devient de ce fait porteur de l'ontologie technique qui en retour lui confère ses propriétés systémiques. Ayant toutes deux des prétentions hégémoniques, technique et capitalisme tendent finalement à se renforcer l'un l'autre. "Ce n'est certainement pas un hasard " écrit Mondoux « si la technique est devenue un réseau systémique global au moment où le capitalisme se déploie dans sa forme transnationale et supranationale " (Ibid., p. 202). Les TIC peuvent donc être considérées comme faisant partie d'un ensemble beaucoup plus vaste et, à ce titre, elles doivent être appréhendées comme autant de composantes du système technicien et au-delà d'un système technico-économique.

\subsection{Du système technique au système économique ou des apports de l'économie politique de la communication}

Les travaux effectués en économie politique de la communication ont justement souligné le rôle central du capitalisme et de ses agents dans le développement des techniques qui nous intéressent. À titre d'exemple, Vincent Mosco, auteur d'une synthèse importante des travaux en économie politique de la communication (1996), s'est intéressé à la fin des années 1990 et au début des années 2000 aux discours sur les "autoroutes de l'information " en analysant ceux-ci comme de nouveaux mythes visant à faire croire que nous participerions collectivement à l'aventure du "cyberespace", alors que le développement de ces dispositifs techniques de communication a servi avant tout les intérêts de certains acteurs sociaux, à commencer par les entreprises transnationales (2003). Et pourtant, on ne parlait pas encore des GAFA (Google, Apple, Facebook et Amazon) qui ont atteint une telle ampleur sur le marché international des industries de la communication qu'ils ont relégué les traditionnels monopoles nationaux au rang de "nains locaux». Les développements historiques de l'internet ont pourtant pendant longtemps échappé aux analyses critiques en économie politique, de par la nature ouverte du processus d'innovation sociotechnique et de par la dimension coopératrice qui motivait la grande majorité des développeur/e/s du réseau qui en étaient également les usagers/ères. Pickering et al. (1996) ont bien montré que la logique de mise en valeur du capital a progressivement pénétré le développement du réseau lorsqu'aux États-Unis les entreprises privées ont été autorisées à investir dans l'exploitation, puis dans la construction de dorsales (backbones), en lieu et place d'organismes comme la National Science Foundation (NSF). Le gouvernement états-unien a alors jugé que le temps était arrivé de modifier son rôle pour non seulement encourager un financement privé, mais également pour privatiser l'architecture du réseau. II s'agissait en 
quelque sorte d'une condition importante pour qu'il puisse être « saisi » comme objet d'étude par les chercheur/e/s en économie politique de la communication. Philippe Bouquillion (2008) soutient que, dorénavant, il est impossible d'aborder de manière critique l'internet et plus largement l'ensemble des TNIC sans les penser en relation avec le développement des industries de la communication. Ces dernières doivent aussi être abordées en rapport à la fois avec les changements dans les industries de contenu (industries de la culture et de l'information) et avec les mutations du capitalisme marquées, entre autres, par la financiarisation.

Certains travaux effectués en économie politique rappellent la pertinence d'aborder des vocables comme ceux de « web 2.0 » ou de « web collaboratif » comme une nouvelle façon de nommer la "société de l'information " (George et Granjon, 2008), et relèvent qu'il s'agit avant tout d'un ensemble de discours d'ordre idéologique visant notamment à intégrer la participation citoyenne dans la production de contenus économiquement valorisables (Bouquillion et Matthews, 2010). Ces auteurs rappellent que l'expression même de "web 2.0 » apparue en 2003 est le plus souvent attribuée à Tim O'Reilly, expert en informatique et entrepreneur, qui cherchait ainsi à relancer le réseau internet en tant que secteur d'activités économiques alors que le tournant du millénaire venait d'être marqué par l'éclatement de la bulle financière créée auparavant autour du réseau informatique. O'Reilly s'est mis à défendre le point de vue selon lequel le salut du « Net » allait reposer sur le modèle «participatif » où l'internaute se devait de quitter le simple statut de consommateur pour celui de " générateur de contenus ». Or, comme l'ont montré Bouquillion et Matthews, la thématique du "web 2.0 » a largement contribué à légitimer les formes prises par le capitalisme depuis la fin de la période fordiste des années 1970. «En effet, les discours relatifs au Web 2.0 reprennent, prolongent et renouvellent d'importants discours sur les industries de la culture et de la communication, ainsi que sur les TIC et leur insertion économique et sociale » (2010, p. 10). On y parle de technique avec des interfaces - enfin conviviales ! - qui vont pouvoir beaucoup mieux décentraliser la prise de parole que ne le faisait jadis le « web 1.0 ( (qui ne se savait pas étiqueté comme tel). II est en revanche beaucoup moins question d'économie dans bon nombre de travaux et, dès lors, les réflexions d'un Christian Fuchs (2014) sur les façons dont les médias socionumériques contribuent plus que jamais à renforcer le capitalisme apparaissent d'autant plus bienvenues (voir aussi George, 2011). On oublie souvent également que les TIC participent à l'établissement des rapports inégaux à une échelle géographique de plus en plus globale (Benchenna, 2012). 
En complément des recherches effectuées en économie politique de la communication, des travaux critiques à orientation sociopolitique ont traité des politiques publiques en la matière. Mais, au cours des dernières années, la pertinence même de ces politiques - et des travaux à orientation critique - a été remise en cause du fait de la difficulté à concevoir et justifier l'application de politiques historiquement légitimées par la souveraineté nationale à l'ère du numérique et de la mondialisation. Nous aurions dorénavant basculé dans un monde d'abondance informationnelle, le pluralisme naîssant automatiquement de la configuration du «web 2.0 », forcément «collaboratif». Dorénavant, quiconque pourrait devenir auteur et diffuser ses œuvres ; ou pour le moins, il y aurait possibilité de diminuer les intermédiaires entre la production et la consommation, par exemple en ce qui concerne le cinéma (Broderick, 2008). Or, la désintermédiation ne se doublerait-elle pas de nouvelles formes de réintermédiation (Bustamante, 2004) ? De plus, si des acteurs comme Google ou Netflix jouent dorénavant un rôle majeur parmi les industries de la communication, ils pénètrent également de plus en plus le champ des industries du contenu (de la culture et de l'information) tout en échappant le plus possible à ces politiques publiques (Bouquillion, 2008).

\subsection{Les études sur les usages des TIC et les questions de l'autonomie et de l'émancipation}

Cela dit, si les travaux en économie politique de la communication ont permis de mieux saisir les tenants et les aboutissants de l'offre capitaliste qui contribuent largement à créer la demande et à structurer les usages des TIC, ils n'ont guère intégré dans les recherches l'étude des usages, mis à part sans doute le cas d'une recherche menée dans les années 1990 par Jean-Guy Lacroix, Gilles Pronovost et Gaëtan Tremblay (Lacroix et al., 1993). D'où la pertinence du lancement d'un programme à la même période malheureusement jamais concrétisé - consacré à une analyse sociopolitique des usages des TIC de la part de Thierry Vedel et d'André Vitalis (Vedel, 1994 ; Vitalis, 1994). Ces deux auteurs s'étaient fixés pour ambition de croiser l'approche microsociologique de l'appropriation, attentive au développement des usages, avec une approche macrosociologique qui, grâce à l'étude des acteurs du champ de l'offre, permettrait de contextualiser d'un point de vue sociohistorique la formation et la structuration des usages. Vedel considérait qu'une sociopolitique des usages impliquerait la réalisation d'études fines des pratiques d'usages grâce à des approches ethno-méthodologiques accordant une place centrale aux entretiens avec les usagers/ères et aux observations de leurs pratiques, tout en s'intéressant aux analyses des discours d'accompagnement et des jeux d'acteurs : "Quelque soit l'angle d'investigation initialement privilégié, l'exigence essentielle sur laquelle repose une sociopolitique des usages est de ne pas réduire l'explication des usages des 
technologies à un principe unique, mais de constamment prendre en compte les interrelations complexes entre outil et contexte, offre et utilisation, technique et social » (1994, p. 32).

Malheureusement, ils ne sont pas allés plus loin. Serait-ce dû au fait que, comme le dit Florence Millerand, il y avait alors une juxtaposition des niveaux macro et micro ? "Comment lier les deux niveaux au sein d'une même analyse ? " s'est-elle posée comme question $(1999$, p. 16). Les travaux qui se sont par la suite inscrits dans cette perspective ont été fort peu nombreux. II importe de souligner d'autant plus ceux de Geneviève Vidal qui a développé la notion de "renoncement négocié " qui tient compte de «l'injonction des technologies interactives disséminées dans la société et les écarts des usagers conscients de leur propre aliénation par les TIC dans la sphère domestique, au travail, en mobilité » (2010, p. 89). Elle conclut d'ailleurs que ceux-ci ont conscience de "la soumission à l'injonction, à l'aliénation de (leurs) propres usages ", le renoncement visant "une négociation, pour soi et par soi, pour faire partie voire participer au réseau de "soutiens à la tyrannie" dans une interdépendance des rapports de force inégale » (Ibid.).

La question de l'autonomie relative dont dispose l'usager/ère, mais aussi le, la concepteur/trice, par rapport au système technico-économique est évidemment centrale dans le cadre des travaux à orientation critique. Or, aussi bien dans le monde anglophone, où des recherches à orientation culturaliste ont été menées dans le cadre des Cultural Studies qu'en milieu francophone, où celles-ci se sont plutôt développées dans celui de la sociologie des usages des TIC (George, 2012), cette question a souvent reposé par le passé sur la mobilisation d'un auteur, Michel de Certeau. Ce dernier a lui-même mis l'accent dans ses travaux consacrés aux pratiques urbaines sur un usager tacticien dans un contexte dominé par les stratégies capitalistes (1980). En faisant justement référence à de Certeau, Pierre Macherey souligne la différence entre les concepts de stratégie et de tactique: "la stratégie est intéressée, sa préoccupation essentielle étant de parvenir à capitaliser des acquis de manière à les métamorphoser en profits ; la tactique, au contraire est désintéressée : elle ne stocke pas, mais en reste au stade d'une production parcellaire, exploratoire, effectuée au coup par coup, donc spontanément, au gré des occasions " (2005). Or, si la sociologie des usages des TIC, et notamment en son sein l'approche de l'appropriation a bien mis en évidence la richesse des pratiques et des représentations des usagers/ères dans toute leur complexité tant individuelle que sociale, Josiane Jouet (2000) se demande si l'accent n'a pas été trop mis de façon générale sur l'autonomie. Fabien Granjon en est même 
venu à constater que certaines recherches consacrées aux usages des TIC relevaient plus d'approches fonctionnalistes que critiques (2004).

Jouët (2000) rappelle que les recherches sur les TIC ont été menées en France dans un contexte post-soixante-huitard où l'autonomie était une valeur connotée très positivement. II apparaît aussi pertinent de rappeler que les ÉtatsUnis étaient marqués à cette époque par la dynamique de la "contre-culture " au sein de laquelle l'autonomie était aussi promue. C'était l'époque du développement de l'informatique en réseau à travers l'invention des microordinateurs, des modems et des babillards (bulletin board services). Dans certains écrits, comme dans l'ouvrage d'Howard Rheingold, Les Communautés virtuelles (1995), il apparaît bien difficile de distinguer analyse distanciée et militantisme. Certains travaux sur les TIC n'ont-ils pas été caractérisés par l'incorporation de positions trop idéologiques favorisant une analyse libérale du sujet/acteur dans la société à partir d'une approche qui se voulait pourtant critique ? Et ne retrouverait-on pas avec le développement du « web 2.0 » et audelà des TNIC ce même type d'analyse? On pense par exemple aux productions d'un auteur comme Henry Jenkins qui estime que la convergence des médias a favorisé l'émergence d'une nouvelle culture participative de la part de personnes ordinaires. Dans son livre Convergence Culture : Where Old and New Media Collide (2006), il a été jusqu'à défendre la thèse selon laquelle les fans et leurs activités tenaient dorénavant un rôle central dans le développement des industries de la culture. Ainsi, entrevoie t-il la possibilité que ceux-ci deviennent de véritables partenaires actifs au carrefour entre culture populaire et culture commerciale dans une société en réseau. Et pour lui, les chercheurs, en tant qu'intellectuels publics, ne doivent plus adopter un positionnement critique par rapport à ce qui advient mais, au contraire, accompagner les changements en cours. II deviendrait en conséquence secondaire de travailler sur l'élaboration de théories critiques au profit du développement d'échanges avec l'ensemble des composantes de nos sociétés, y compris les entreprises, qui sont impliquées dans les changements en cours (Jenkins, 2007). Or, si une excellente connaissance du terrain est évidemment pertinente à des fins d'analyse, il n'en apparaît pas moins que la démarche de recherche, surtout critique comme nous l'avons présenté précédemment, possède certaines spécificités et exigences.

Ainsi, il ne s'agit pas de considérer que, de prime abord, les mutations annoncées autour des TIC ou des TNIC et notamment du « web 2.0 » existent ou n'existent pas. Des auteurs comme Jenkins mettent incontestablement l'accent sur des phénomènes intéressants. Et en tant que chercheur critique, nous ne pouvons que porter une attention aux changements en cours qui favoriseraient éventuellement une plus large expression, une augmentation de l'empowerment et en dernier ressort l'augmentation de la capacité d'agir de tout 
un chacun à des fins de participation à notre devenir, tant individuel que collectif. Mais il importe en même temps de se souvenir de ce que nous dit Cornelius Castoriadis, à savoir que "le projet d'autonomie individuelle et collective, la lutte pour l'émancipation de l'être humain, aussi bien intellectuelle et spirituelle qu'effective dans la réalité sociale» $(1996$, p. 90) se retrouve toujours confrontée au projet «capitaliste, démentiel, d'une expansion illimitée d'une pseudo-maîtrise pseudo-rationnelle, qui depuis longtemps a cessé de concerner seulement les forces productives et l'économie pour devenir un projet global (et pour autant encore plus monstrueux), d'une maîtrise totale des données physiques, biologiques, psychiques, sociales, culturelles » (Ibid.).

Il apparaît en conséquence impossible de traiter de la question des usages des TIC ou des TNIC dans les transformations sociales à l'œuvre sans tenir compte des rapports entre les différents agents sociaux. Et à ce sujet, il faut souligner par exemple le travail emblématique effectué par plusieurs chercheur/e/s au fil des années, recensé dans un ouvrage collectif ayant pour titre Inégalités numériques. Clivages sociaux et modes d'appropriation des TIC (Granjon et al., 2009). Où l'on y retrouve la variété des modes de domination, des rapports de pouvoir que l'on peut saisir tant d'un point de vue macro que micro. C'est d'ailleurs l'occasion de souligner deux éléments centraux de notre réflexion : premièrement, le capitalisme constitue plus que jamais une source essentielle des rapports de pouvoir. Ainsi, faut-il entendre celui-ci non seulement comme un mode de production, mais au-delà comme un mode de reproduction sociale qui repose fondamentalement sur la centralité de la rationalité économique, l'économique tendant à subsumer le social. Deuxièmement, d'autres formes de domination perdurent évidemment, dont certaines plus anciennes que le capital, comme le patriarcat, et méritent aussi toute notre attention.

Cela dit, il n'est pas question pour autant de ne considérer les rapports entre TIC et société uniquement du point de vue des rapports de domination, des rapports de pouvoir. II importe aussi d'envisager les possibles liés aux TIC. Et ceci nous renvoie à ce que nous avancions précédemment en ce qui concerne le terme «critique » (Horkheimer, 1996). Cela rappelle également les propos de Jean-Marc Fontan qui mentionne que toute pensée critique «dénonce l'inacceptable tout en permettant ou en proposant des correctifs. La pensée critique vise fondamentalement la réalisation d'un projet de transformation positive de la société » $(2000$, p. 66). Pour notre part, alors qu'Horkheimer adoptait le possible au singulier, nous préférons employer à dessein le pluriel afin de prendre position en faveur d'une lecture de l'histoire non déterministe. Nous rejoignons ici la position d'Haud Gueguen (2014) qui s'est fixée pour 
objectif d'interroger cette catégorie du possible en s'intéressant aux études sur le numérique.

Ses interrogations s'avèrent d'autant plus pertinentes que, comme elle le dit elle-même, les travaux qui traitent le plus souvent des potentialités des TIC en termes d'émancipation soit tendent à réduire le possible à sa composante technique en faisant abstraction du social et des rapports de force qui le composent, soit sont tentés de faire fi de toute perspective historique en favorisant une approche présentiste pour laquelle chaque nouvel objet technique est appréhendé sur une échelle de court, voire de très court terme comme une révolution. Ceci rappelle les préoccupations d'Armand Mattelart (2014) concernant l'importance d'intégrer le temps long dans l'analyse des phénomènes et processus de communication. On retrouve également ici la mise en garde de Tremblay: "dans le secteur des communications comme en d'autres domaines, les changements les plus profonds qui accompagnent les innovations techniques ne peuvent être correctement appréhendés qu'à long terme. Les recherches empiriques à brève échéance, si sophistiquées et si élaborées soient-elles, constituent des instruments bien limités pour identifier les tendances de fond. » $(2007$, p. 121).

\section{De la pertinence de tenir compte de deux autres dimensions: le temps long et l'environnement}

Deux auteurs attirent justement notre attention sur la pertinence des perspectives de recherche intégrant le temps long ainsi qu'une certaine matérialité de la technique au sein des perspectives critiques. Pour Harold Innis, parler de TIC revient à parler de "médias " ou bien encore de "moyens de communication». En fait, le chercheur canadien, parfois considéré comme le "père fondateur " des études en communication dans ce pays, s'intéresse fondamentalement à l'ensemble des moyens matériels et intellectuels mobilisés par une civilisation afin de transmettre et de stocker dans le temps et pour les générations à venir le savoir. II peut donc s'agir de la tablette d'argile, du papyrus, des bibliothèques, de la télévision, etc. Jack Goody mobilise de son côté le concept de "technologies intellectuelles » et en donne la justification suivante : "Même si l'on ne peut raisonnablement pas réduire un message au moyen matériel de sa transmission, tout changement dans le système des communications a nécessairement d'importants effets sur les contenus transmis » (1979, p. 46). Leurs pensées présentent à la fois des points communs et des différences comme le montre Oumar Kane (2013). Mais ce qui nous intéresse plus particulièrement ici, c'est qu'ils partagent une " perspective agonistique commune puisque les médias ou les modes de communication induisent une dissymétrie parmi les acteurs sociaux et dans les rapports de 
force en fonction des biais qui leur sont inhérents (Innis, 1968, 1972) ou des exclusions qu'elles induisent (Goody, 1979, 1986). ” (Kane, 2013).

L'illustration suivante est intéressante à ce sujet. Dans "L'Oiseau de Minerve » (1983), l'accent est mis par Innis sur la civilisation mésopotamienne située dans la vallée de deux fleuves, le Tigre et de l'Euphrate, une vallée riche en argile, environ 3000 ans avant notre ère. L'argile pût servir de support à la communication à partir du moment où un instrument d'écriture approprié fut mis au point (un roseau taillé en pointe) et lorsqu'un système symbolique, un alphabet fut inventé (l'écriture cunéiforme). Le moyen de communication, au sens innissien du terme, était donc composé des tables d'argile, du roseau et de l'écriture cunéiforme et il avait alors pour caractéristique principale d'exiger, note Innis, une grande dextérité afin d'être utilisé correctement. En conséquence, ainsi que l'explique Paul Attallah (2001, p. 252), il était réservé à une petite élite qui disposait largement de temps, à savoir les prêtres. On voit bien à travers cet exemple comment un moyen de communication, en l'occurrence la combinaison entre l'argile, le roseau et l'écriture cunéiforme, a favorisé l'émergence d'un monopole du savoir reposant sur les religieux. Plus généralement, Innis aboutit à l'idée selon laquelle ceux qui, dans une société donnée, contrôlent le savoir par l'entremise des technologies dominantes (membres de l'élite érudite, gouvernementale, religieuse ou professionnelle) contrôlent aussi la réalité, en ce sens qu'ils sont en mesure de définir quel savoir est légitimé. De la sorte, les monopoles du savoir encouragent la centralisation du pouvoir. Innis fait donc des liens entre plusieurs caractéristiques d'une époque : les positions sociales des différentes composantes de la société, la disponibilité de certains moyens de communication, la maîtrise de ceux-ci et le développement des savoirs. Comme le dit Attallah ( $\mathrm{lbid}$.), le monopole du savoir dépend non seulement de la maîtrise des médias, mais aussi d'une constellation d'autres facteurs sociaux d'ordre économique, politique, religieux. Pour Attallah, le modèle d'Innis est donc un modèle à causalité multiple, et non pas médiacentrique, même s'il accorde une place centrale à ceux-ci. Des auteurs comme Goody et Innis ont le mérite de mettre l'accent sur plusieurs éléments mentionnés plus tôt dans notre texte : les TIC font partie d'un ensemble plus vaste de techniques et doivent donc être appréhendées comme des artéfacts, mais elles présentent également des spécificités de par le fait même qu'elles constituent des moyens de communication au sens fort du terme. Comme le dit Tremblay, "les travaux d'Innis et de McLuhan ont établi de façon définitive que chaque média présente une configuration spécifique de possibilités et de contraintes que toute théorie des communications doit prendre en compte » (2007, p. 122). 
Enfin, si tous les travaux mentionnés dans ce texte ont mis l'accent sur les questions d'ordre économique, politique, culturel, social et technique, ils n'ont pas ou guère intégré les enjeux d'ordre environnemental. Ce n'est guère étonnant étant donné que nous nous sommes concentré uniquement sur une épistémologie occidentale. Pourtant, tant les stratégies industrielles que les pratiques culturelles mobilisant des TIC constituent des sources de pollution considérables, que l'on fasse référence ici au gaz à effet de serre produit par l'utilisation massive de serveurs ou bien aux déchets causés par l'abandon de tous les appareils, des téléviseurs aux ordinateurs en passant par les imprimantes, jugés de plus en plus rapidement obsolètes. II serait donc d'autant plus judicieux d'élargir la pensée critique consacrée aux TIC à cette question environnementale, ainsi que nous y invite Dominique Carré (2012). À plus ou moins long terme, c'est la survie de notre espèce, du moins telle que nous la connaissons, qui est en jeu. Pour envisager au contraire une nouvelle étape dans l'histoire de l'humanité, ne serait-il pas crucial de repenser non seulement nos rapports entre humains, entre humains et technique mais également ceux que nous entretenons avec la nature ? II nous semble à ce sujet que d'autres épistémologies, à commencer par l'épistémè autochtone amérindienne aurait beaucoup à nous apprendre sur les liens symbiotiques qui nous unissent avec notre Terre-mère, par exemple à partir de la prise en compte du fait que rien ne nous permet de légitimer la domination des espèces par l'une d'entre elles qui serait considérée comme supérieure aux autres (Sioui, 1999).

\section{Références bibliographiques}

ADORNO T., 1964, «L'industrie culturelle », Communications, n³, pp. 12-18.

ABBATE J., 1999, Inventing the Internet, Cambridge (Mass.), MIT Press.

ATTALLAH P., 2001, Théories de la communication. Histoire, contexte, pouvoir, Montréal, Université du Québec, Télé-Université.

BALIBAR É., 1992, Les frontières de la démocratie, Paris, La Découverte.

BENCHENNA A., 2012, "Les Tics dans les pays des Suds: 40 années de recherche. 1970-2010", tic\&société, vol. 5, n²-3, http://ticetsociete.revues.org/1130, dernière consultation : 18/05/2014.

BOUQUILLION P., 2008, Les industries de la culture et de la communication. Les stratégies du capitalisme, Grenoble, Presses universitaires de Grenoble.

BOUQULLION P. et J. MATTHEWS, 2010, Le Web collaboratif. Mutations des industries de la culture et de la communication, Grenoble, Presses Universitaires de Grenoble. 
BRODERICK P., 2008, "Welcome to the New World of Distribution ", http://www.peterbroderick.com/writing/writing/welcometothenewworld.html, dernière consultation : 18/05/2014.

BUSTAMANTE E., 2004, "Cultural industries in the Digital Age: some provisional conclusions ", Media, Culture and Society, vol. 26, nº , pp. 803-820.

CARRÉ D., 2013, "Approche critique et techniques numériques d'infocommunication: Vers la prise en compte d'une nouvelle dimension ? ", dans O. KANE et É. GEORGE (dir.), Où (en) est la critique en communication ?, Montréal, pp. 61-73, http://gricis.uqam.ca/activites/colloques/article/ou-en-est-lacritique-en, dernière consultation : 18/05/2014.

CASTELLS M., 1998, L'ère de l'information. Vol. 1, La société en réseaux, Paris, Fayard.

CASTORIADIS. C., 1996, La montée de l'insignifiance. Les carrefours du labyrinthe. Tome IV, Paris, Le Seuil.

CERTEAU M. de, 1980, L'Invention du quotidien, tome 1 Arts de faire, Paris, Gallimard.

ELLUL J., 1954, La technique où l'enjeu du siècle, Paris, Armand Collin.

ELLUL J., 1973, Les nouveaux possédés, Paris, Fayard.

ELLUL J., 1977, Le système technicien, Paris, Calmann-Lévy.

ELLUL J., 1988, Le bluff technologique, Paris, Hachette.

FUCHS C., 2014, Social Media : A Critical Introduction. Londres, Sage.

GEORGE É. et F. GRANJON, 2008, Critiques de la société de l'information, Paris, L'Harmattan.

GEORGE É., 2011, "L'intrusion de Google dans la vie privée au cœur des mutations du capitalisme », Terminal, n 108-109, pp. 91-108.

GEORGE É., 2012, "L'étude des usages des TIC au prisme de la recherche critique en communication », dans G. VIDAL (dir.), La sociologie des usages à l'heure de l'intensification des technologies de l'information et de la communication et des réseaux numériques, Paris, Hermes Science, pp. 25-62.

GEORGE É., 2014, “Éléments d'une épistémologie critique en communication. Au carrefour et au-delà de l'école de Francfort, des Cultural Studies et de l'économie politique de la communication ", dans É. GEORGE et F. GRANJON 


\section{Éric GEORGE}

(dir.), Critique, sciences sociales et communication, Paris, Mare\&Martin, à paraître.

GOODY Jack, 1979, La raison graphique. La domestication de la pensée sauvage, Paris, Les Editions de Minuit.

GOODY J., 1986, La logique de l'écriture. Aux origines des sociétés humaines, Paris, Armand Colin.

GRANJON F., 2004, "De quelques éléments programmatiques pour une sociologie critique des usages sociaux des TIC ", dans S. HADJ-ALI (dir.), Les rapports société-technique du point de vue des sciences de l'homme et de la société, Intervention, Journée d'étude organisée par le LARES-Université de Rennes 2.

GRANJON F., B. LELONG et J.-P. METZGER (dir.), 2009, Inégalités numériques : clivages sociaux et modes d'appropriation des TIC, Paris, Lavoisier.

GUEGUEN H., 2014, "La critique et le possible : le rôle de la catégorie de possible dans la critique des TNIC », dans É. GEORGE et F. GRANJON (dir.), Critique, sciences sociales \& communication, Paris, Mare\&Martin, à paraître.

HORKHEIMER M., 1996, Théorie traditionnelle et théorie critique, Paris, Gallimard.

INNIS H., 1950, Empire and Communications, Toronto, University of Toronto Press, nouvelle édition : 1972.

INNIS H., 1951, The Bias of Communication, Toronto, University of Toronto Press, nouvelle édition : 1968.

INNIS H., 1983, "L'oiseau de Minerve », Communication Information, vol. 5, n²3, pp. 267-297.

JENKINS H., 2006, Convergence Culture : Where Old and New Media Collide, New York, NYU Press.

JENKINS H., 2007, «The Moral Economy of Web 2.0: Reconsidering the Relations between Producers and Consumer, Conférence de l'AolR", Vancouver, http://blip.tv/anthrovlog/aoir-2007-jenkins-talk-515391, dernière consultation : 18/05/2014.

JOUËT J., 2000, « Retour critique sur la sociologie des usages », Réseaux, vol. 18, $\mathrm{n}^{\circ} 100$,

pp. 487-521, http://www.persee.fr/web/revues/home/prescript/article/reso 075179712000 num 18100 2235, dernière consultation : 18/05/2014.

KANE O, 2013, « Les usages des TIC entre analyse sociotechnique et théories de l'appropriation : état de la littérature », dans G. BERTHIN MADEBE (dir.), Les 
enjeux de la communication, Libreville, Presses universitaires du Gabon, pp. 2342.

KANE O. et É. GEORGE (dir.), 2013, Où (en) est la critique en communication ?, Actes du colloque organisé par le CRICIS, Montréal, les 7 et 8 mai 2012, 371 p.,http://gricis.uqam.ca/activites/colloques/article/ou-en-est-la-critique-en.

LACROIX J.-G., 1998, "Sociologie et transition millénariste: entre l'irraison totalitaire du capitalisme et la possibilité-nécessité de la conscientivité ", Cahiers de recherche sociologique, $\mathrm{n}^{\circ} 30, \mathrm{pp} .79-152$.

LACROIX J.-G., G. PRONOVOST et G. TREMBLAY, 1993, "La mise en place de l'offre et la formation des usages des NTIC ", Cahiers de recherche sociologique, $\mathrm{n}^{\circ} 21$, pp. 79-122.

MACHEREY P., 2005, "Michel de Certeau et la mystique du quotidien ", Séminaire de l'unité mixte de recherche Savoirs textes langage, année 20042005, http://stl.recherche.univlille3.fr/seminaires/philosophie/macherey/macherey20042005/macherey060420 05.html, dernière consultation : 18/05/2014.

MARCUSE H., 1968, L'homme unidimensionnel. Essai sur l'idéologie de la société avancée, Paris, éditions de Minuit.

MATTELART A., 2014, De la difficulté à penser l'international au regard de l'histoire : leçons d'un itinéraire intellectuel, dans GEORGE É. et F. GRANJON (dir.), Critique, sciences sociales \& communication, Paris, Mare\&Martin, à paraître.

MILLERAND F., 1999, "Usages des NTIC : les approches de la diffusion, de l'innovation et de l'appropriation (2e partie) ", COMMposite, v1999.1, http://commposite.org/index.php/revue/article/viewArticle/98, dernière consultation : 18/05/2014.

MONDOUX A., 2007, Technique, individuation et (re)production sociale. La musique numérique MP3, thèse de doctorat en sociologie, Montréal, Université du Québec à Montréal.

MORIN E., 2004, "Au-delà de la globalisation et du développement, sociétémonde ou empire-monde ? ", La revue du MAUSS, http://www.cairn.info/revuedu-mauss-2002-2-page-43.htm, dernière consultation : 18/05/2014.

MOSCO V., 1996, The Political Economy of Communication. Thousand Oaks (CA), Sage. 


\section{Éric GEORGE}

MOSCO V., 2003, « Brand New World? Globalization, Cyberspace and the Politics of Convergence », dans G. TREMBLAY et B. MIĖGE (dir.), 2001 Bugs. Globalism and Pluralism, Québec, Les Presses de l'Université Laval, pp. 31-61.

OUELLET M., 2009, Le capitalisme cybernétique dans la société globale de l'information: une approche culturelle de l'économie politique internationale, thèse de doctorat en sciences politiques, Ottawa, Université d'Ottawa.

PICKERING J. M., R. E. GRINTER et J.-L. KING, 1996, « Grandeur et décadence d'Arpanet. La saga de Netville, cité champignon du cyberespace ", Réseaux, vol. 14 $\mathrm{n}^{\circ} 77$, pp. 9-35, http://www.persee.fr/web/revues/home/prescript/article/reso 075179711996 num 1477 3733, dernière consultation : 18/05/2014.

PROULX S. et M. SÉNÉCAL, 1995, "L'interactivité technique, simulacre d'interaction sociale et de démocratie ? ", TIS, vol. 7, n², pp. 239-255.

RANCIERE J., 2004, Le maître ignorant, Paris, $10 \times 18$.

tic\&société, 2010, Interactivité et lien social, vol. 4, $\mathrm{n}^{\circ} 1$, http://ticetsociete.revues.org/750, dernière consultation : 18/05/2014.

RHEINGOLD H., 1995, Les communautés virtuelles, Paris, Addison-Wesley.

SIOUI G., 1999, Pour une histoire amérindienne de l'Amérique, Québec, Presses de l'Université Laval.

THOMPSON J. B, 1987, « Langage et idéologie », Langage et société, n³9, pp. 730.

TREMBLAY G., 2007, « De Marshall McLuhan à Harold Innis ou du village global à l'empire mondial ", tic\&société, vol. 1, $n^{\circ} 1$, pp. 104-129, http://ticetsociete.revues.org/222, dernière consultation : 18/05/2014.

VEDEL T., 1994, "Sociologie des innovations technologiques des usagers: introduction à une socio-politique des usages ", dans A. VITALIS (dir.), Médias et nouvelles technologies. Pour une socio-politique des usages, Rennes, Apogée, pp. 13-34.

VIDAL G., 2010, Le renoncement négocié. Pour une analyse dialectique des usages des technologies interactives, Mémoire d'habilitation à diriger des recherches, Bordeaux, Université Michel de Montaigne.

VITALIS A., 1994, "La part de citoyenneté dans les usages », dans A. VITALIS (dir.), Médias et nouvelles technologies. Pour une socio-politique des usages, Rennes, Apogée, pp. 35-43. 